\title{
LESSONS LEARNT FROM THE AUTOPSY FINDINGS OF COVID-19: HERE THE DEAD TEACH THE LIVING.
}

\section{Pathology}

Dr. Bhanu Sharma

M.B.B.S.Resident, Deptt. of Pathology, SGT Medical College \& University, Gurugram, Haryana.

\section{Dr. Namita Bhutani*}

M.B.B.S, M.D, D.N.B. Assistant Professor, Deptt. of Pathology,North DMC Medical Dr. Sunil Arora College \& Hindu Rao Hospital, Delhi. * Corresponding Author

Dr. Sunil Aroram, Haryana.

Dr. Sachet Dawar

\section{Dr. Raj Kumar} Gupta

\section{Dr. Pooja Poswal}

M.B.B.S, M.D. Assistant Professor, Deptt. of Pulmonary Medicine, SGT Medical College \& University, Gurugram, Haryana.

M.B.B.S, M.D., Assistant Professor, Deptt. of Pathology, SGT Medical College \& University, Gurugram, Haryana.

M.B.B.S., Resident,Deptt. of Pathology, SGT Medical College \& University, Gurugram, Haryana.

\section{ABSTRACT}

On 31 December 2019, first case of corona virus was reported from China. Since then, the current outbreak of a novel coronavirus (2019^nCoV) has been declared as a global emergency. This eventually has lead to an increase in the number of coronavirus disease 2019 (COVID-19)-related deaths at autopsy. The Royal College of Pathologists (RCP) has responded to this concern with the release of autopsy practices relating to COVID-19. Data on pathologic changes seen in COVID-19 are scarce. To gain insights about the pathology of the disease progression and fatality, a review was performed on the autopsy findings of these patients. In this review, we discuss briefly about the pathology and pathogenesis of novel corona virus. This analysis may help understand pathogenesis and clinical outcomes of this life-threatening illness.

\section{KEYWORDS}

Coronavirus; Immunopathology; Pathogenesis; Respiratory System; Zoonotic Disease.

\section{INTRODUCTION}

In the end of December 2019, local health organisations in Wuhan, Hubei Province, China reported few patients with pneumonia of unknown aetiology [1]. And in early January 2020, the causative agent of this pneumonia was identified as a novel coronavirus, independently, by many laboratories in China. World Health Organization (WHO) named the causative virus as novel coronavirus 2019 (2019"nCoV) [2]. By 24 $4^{\text {th }}$ April, 2020, according to the daily report of WHO, the epidemic of 2019-nCoV had 26,26,321 confirmed cases and 181938 death in world. Before this epidemic, the $21^{\text {st }}$ century has already observed outbreaks caused by two other highly pathogenic corona viruses, namely, the severe acute respiratory syndrome coronavirus (SARS- $\mathrm{CoV}$ ) and the Middle East respiratory syndrome corona virus (MERS-CoV). These outbreaks resulted from zoonotic corona viruses and they resulted in high mortality and morbidity in humans $[3,4]$.

The 2019-nCoV, SARS-CoV and MERS-Co, all these viruses have several similarities in clinical presentations, which vary from asymptomatic infection to severe acute respiratory syndrome $[5,6]$. Therefore, our current knowledge of the pathology and autopsy findings in COVID-19 patients may provide clues for ongoing and future research projects regarding the investigations of this fatal disease. Here, we have tried to apply current understanding of the pathogenesis and autopsy findings in COVID-19 patients for the characterization of $2019 \cdot \mathrm{nCoV}$.

\section{Autopsy Findings in Various Organs}

\section{Kidneys}

A study was conducted in China by $\mathrm{Su}$ et al, highlighted renal histopathological changes in postmortems of patients with COVID19. They stated hypoxia, abnormal coagulation and rhabdomyolysis are the major factors contributing to kidney injury in COVID-19 patients. Only 9 of the presented patients had a pre-morbid urinalysis. Seven of nine presented cases had proteinuria and 6 of 9 had haematuria. Two patients had acute pyelonephritis and 5 patients (19\%) required continuous renal replacement therapy [7]. In COVID19 autopsies all reported cases were found to have mild to severe acute renal tubular injury (Figure 1a \& 1b)

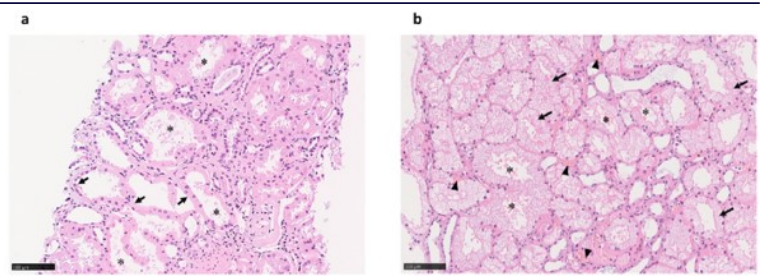

Figure 1 Proximal tubules showed (a) loss of brush border and (b) vacuolar degeneration (arrows), with debris composed of necrotic epithelium in tubular lumens (asterisks). Erythrocyte aggregates obstructing peritubular capillaries were frequently present (arrowheads).

Proximal tubular brush borders were lost and this was one of the characteristic feature of acute tubular injury with coagulative necrosis, vacuolar degeneration (non-isometric in most cases), (4 cases), hemosiderin granules within tubular cytoplasm and pigmented casts within tubular lumens. Deposition of hemosiderin was present in four cases. Evidence of glomerular ischemia was also seen. No significant finding was seen on Immunofluorescence (performed on the paraffinembedded tissue), except for a single reported case of IgA nephropathy. Fibrin tactoids or platelet aggregates were not observed in any COVID19 cases. The receptor for SARS-CoV2, ACE2, was found to be overexpressed in parenchyma of kidney sections of COVID19 (Figure2).
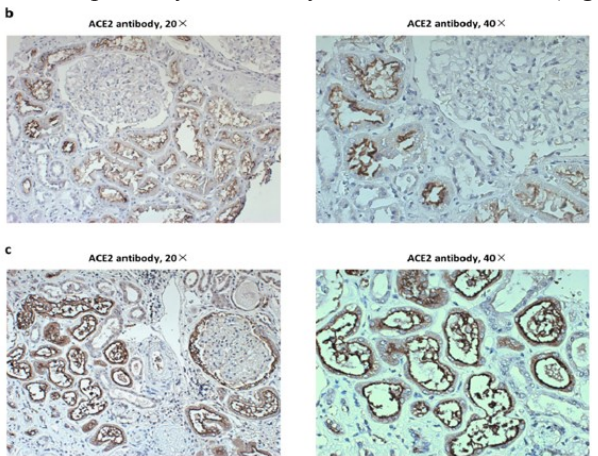

Figure 2 Immunostaining of paraffin-embedded kidney tissue from International Journal of Scientific Research 
patients with coronavirus disease 2019 (COVID-19). (b,c) Angiotensin-converting enzyme II (ACE2) stained mainly proximal tubules in (b) non-coronavirus disease 2019 case, with (c) strong proximal tubular staining and parietal epithelial cell staining with occasional weak podocyte staining in some COVID-2019 cases. (d) Indirect immunofluorescent staining with anti-severe acute respirator syndrome coronavirus (SARS CoV) nucleoprotein antibody.

Positive immunofluorescence staining for SARS-CoV2 antibody within tubular epithelial cells was also present within 3 of 6 cases. In all decedents, hypertensive-related vascular changes were seen, and were found to be moderate to severe in 18 patients while platelet rich thrombi and red blood cell fragmentation were not identified in any case.

\section{Lungs}

Tian $\mathrm{S}$ et al conducted a study to analyse post mortem core biopsies of Cov2 patients and they documented that in lungs microscopic changes varied among all of the four cases, but diffuse alveolar damage (DAD) was present in all cases. First three cases showed vascular congestion and formation of hyaline membrane, which suggested, that this is an acute-phase component reaction (Figure $3 \mathrm{a}-\mathrm{c}$ ).

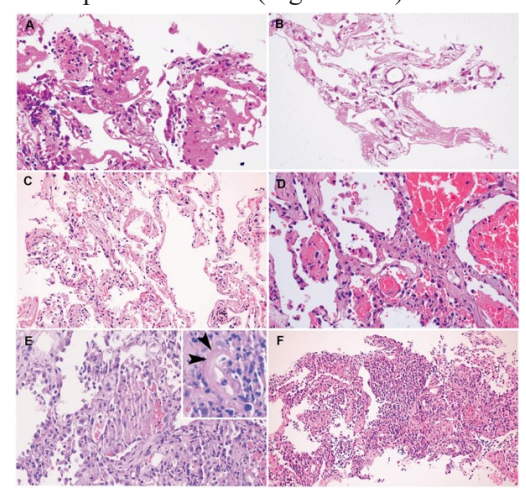

Figure 3 Histologic changes in the lungs. A: thick hyaline membrane mixed with desquamative pneumocytes and mononuclear inflammatory cells. B: more delicate hyaline membranes without evident inflammatory infiltration. C: focal hyaline membrane, type II pneumocyte hyperplasia, and mild interstitial thickening. D: alveolar spaces were filled with red blood cell exudation, and small fibrin plugs seen in adjacent alveoli. E: Organization with intra-alveolar fibroblasts mixed with fibrin and inflammatory cellular infiltration. Diffuse type II pneumocyte hyperplasia in the background (inset: fibrinoid vascular necrosis, arrow heads). F:Changes of bronchopneumonia with prominent neutrophilic infiltration filling up alveolar spaces.

Occasionally, scant inflammatory cell infiltrate was found. In addition to these findings, Case 1 also exhibited formation of syncytial giant cells and pneumocyte injury with focal sloughing (Figure 3a). Case 2 exhibited no prominent inflammatory cellular infiltratate (Figure 3b) and Case 3 exhibited focal interstitial thickening of the basement membrane (Figure 3c). More advanced changes were seen in case 4 . Large areas of intra-alveolar haemorrhages and intra-alveolar fibrin formation were observed nearby in addition to remnants of hyaline membranes in few of the airspaces (Figure 3d). In addition to these findings, the alveolar wall contained increased stromal cells, fibrin and infiltration by mononuclear inflammatory cells. Together with type II pneumocyte hyperplasia, these changes led to interstitial thickening (Figure 3e). There was evidence of small vessels fibrinoid necrosis as well (Figure 3e, inset). Moreover, consolidation was seen and this was evident by abundant intra-alveolar neutrophilic infiltrate, consistent with bronchopneumonia of superimposed bacterial infection [8].

According to a study by Fox et al, the lungs were extensively sampled, both across peripheral regions and central regions of each lobe bilaterally. Histological examination revealed bilateral diffuse alveolar damage with mild-to-moderate lymphocytic infiltrate, which was composed of a mixture of CD4+ and CD8+ lymphocytes (Figure $4 a)$, located mainly in the interstitial spaces and around larger bronchioles. CD4+ lymphocytes could be seen in aggregates around small vessels, some of which appeared to contain platelets and small thrombi. In all but one case, foci of haemorrhage were also present. Desquamated type 2-pneumocytes with apparent viral cytopathic effect consisting of cytomegaly and enlarged nuclei with bright, eosinophilic nucleoli had been found to be present within these alveolar spaces (Figure 4b). Viral inclusions were also documented. Trichrome stain highlighted, scattered hyaline membranes as well as fibrin deposition (Figure 4c). This was consistent with diffuse alveolar damage. The alveolar capillaries were remarkably thickened, with surrounding edema, and fibrin thrombi were present within the capillaries and small vessels (Figure 4d). A notable finding was the presence of CD61+ megakaryocytes (Figure 4e) possibly representing resident pulmonary megakaryocytes, with significant nuclear atypia and hyperchromasia. These cells were present within the alveolar capillaries (Figure 4f). The fibrin and platelets present within small vessels also appeared to aggregate inflammatory cells, with entrapment of numerous neutrophils. In immunosupressed patient, there was evidence of focal acute inflammatory infiltrate possibly consistent with secondary infection. No significant neutrophilic infiltrate was identified within airways or the interstitium to suggest secondary infection in other cases [9].

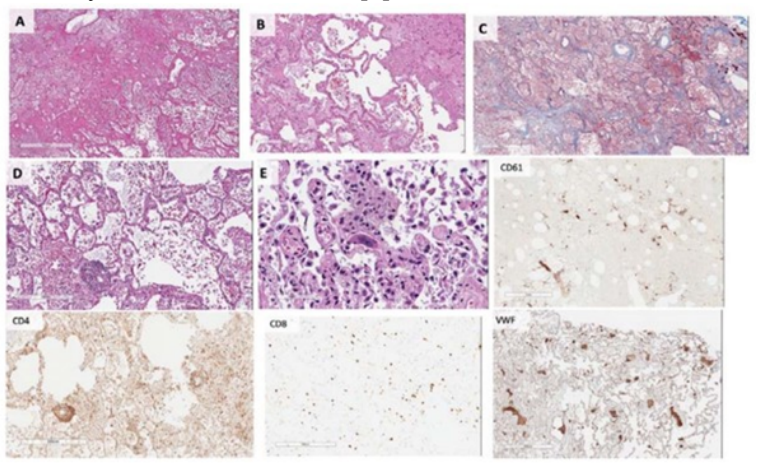

Figure 4 Histologic changes in the lungs. A: bilateral diffuse alveolar damage with a mild-to-moderate lymphocytic infiltrate. B: Desquamated type 2-pneumocytes with apparent viral cytopathic effect consisting of cytomegaly and enlarged nuclei with bright, eosinophilic nucleoli. C: Scattered hyaline membranes could be seen, as well as fibrin deposition, highlighted by trichrome stains. D: The alveolar capillaries were notably thickened, with surrounding edema, and fibrin thrombi. E: presence of CD61+ megakaryocytes representing resident pulmonary megakaryocytes, with significant nuclear atypia and hyperchromasia. F: These cells were located within alveolar capillaries, and could be seen in association with actively producing platelets.

In a study by Zhexu et al, histological examination of lungs revealed bilateral diffuse alveolar damage with cellular fibromyxoid exudates. The right lung showed desquamation of pneumocytes and formation of hyaline membrane, indicating acute respiratory distress syndrome. The left lung showed pulmonary oedema with hyaline membrane formation, suggestive of early-phase ARDS. Interstitial mononuclear inflammatory infiltrates, dominated by lymphocytes, were seen in both of the lungs. Multinucleated syncytial cells with atypical enlarged pneumocytes characterised by large nuclei, amphophilic granular cytoplasm, and prominent nucleoli were identified in the intra-alveolar spaces, showing viral cytopathic-like changes. No obvious intranuclear or intracytoplasmic viral inclusions were identified in any of the tissues [10].

B Henley et al conducted a study and find out that a 50-years-old man died from severe COVID-19 infection. And autopsy was performed and histopathological findings were noted. Ten samples of post mortem biopsy were taken, and a description of the gross post-mortem findings was not given, although multiple ground glass opacities were noted on chest X-ray. The microscopic findings included in the study were diffuse alveolar damage with exudates formation [10]. The inflammation comprised predominantly of lymphocytes and multinucleated giant cells alongside large atypical pneumocytes. Although no definitive viral inclusions were noted[11]. The histopathological features were very similar to those observed in SARS and MERS-corona virus infections.

Carsana et al revealed heavy, congested, boggy and oedematous organs, with spotty involvement. On histology, the features of DAD were found, corresponding to those observed in the exudative and early/intermediate proliferative phases of the disease. Both phases overlapped in the different areas of the lungs. The fibrotic phase was rarely observed, this could be due the short duration of the disease. Moreover, five patients also had bacterial (4) and fungal (1) abscesses [12] 
Liver

Tien et al [8] conducted a study through post mortem core biopsies of liver. They stated that the liver revealed mild sinusoidal dilatation, which is otherwise a common nonspecific change in terminally ill patients. Apart from these changes, focal macrovesicular steatosis, nuclear glycogen accumulation in hepatocytes and dense atypical small lymphocytes in portal tracts were also seen (Figure 5).

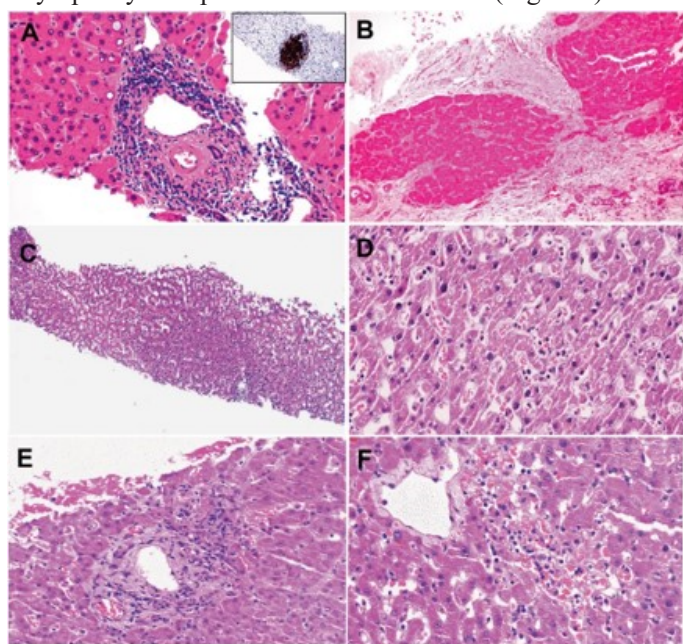

Figure 5 Pathological findings in liver A: Dense portal infiltration by atypical small lymphocytes (insert: CD20 immunostaining), and focal glycogenated nuclei in hepatocyte. B: Cirrhotic nodules with thick fibrosis, C: Mild sinusoidal dilatation with increased lymphocytic infiltration, D: Higher power view showing sinusoidal lymphocytes. E: Focal hepatic necrosis in periportal zone. F: Focal centrilobular hepatic necrosis.

\section{Heart}

Fox et al stated that cardiomegaly was the most common gross finding with right ventricular dilatation. There was no evidence of myocyte necrosis. The histopathology was remarkable. However, scattered individual cell myocyte necrosis was evident in each speciemen examined (Figure 6)

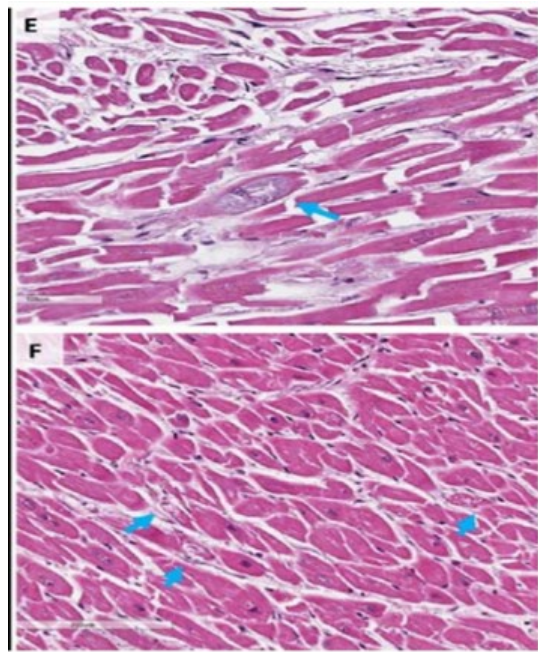

Figure 6 Histologic Findings in the heart. A: areas of myocyte necrosis. B: lymphocytes were found adjacent to degenerating myocytes (but not surrounding).

In few areas lymphocytes were found adjacent to degenerating myocytes (but not surrounding). Whether this was an early manifestation of a viral myocarditis is not known, but there was no significant lymphocytic inflammatory infiltrate that is typical of viral myocarditis. Chen et al stated that pericytes may be infected by the SARS-CoV-2 virus. And this virus can cause capillary endothelial cell/microvascular dysfunction which may cause individual cell necrosis. There was no clear viral cytopathic effect found on light microscopy, but the possibility of direct viral infection of myocytes cannot be ruled out [9].
Tian S et al studied cardiac biopsies in two cases and found that their endocardium and myocardium did not show any signs of inflammation. There were diverse tiers of focal edema, interstitial fibrosis and myocardial hypertrophy. These findings probable constitute pre-existing modifications related to patients underlying diseases, together with hypertension-associated myocardial hypertrophy and past ischemic injury [8].

\section{DISCUSSION}

The autopsy findings in this article are representative of the various previous autopsy studies conducted on cases of COVID-19.

Tian S et al conducted a study of coronavirus through post mortem core biopsies. All cases were consistent with DAD and most cases revealed vascular congestion and hyaline membrane formation. This was suggestive of an acute-phase component with more advanced cases showing large areas of intra-alveolar haemorrhages and fibrin cluster formation in addition to remnants of hyaline membranes in few airspaces. There was also evidence of consolidation by abundant intraalveolar neutrophilic inflammatory infiltrate, consistent with bronchopneumonia of a superimposed bacterial infection [8]. In a study conducted by Fox et al, the lungs showed bilateral diffuse alveolar damage with a comparatively mild-to-moderate lymphocytic inflammatory infiltrate, composed of a mixture of CD4+ and CD8+ lymphocytes located predominantly in the interstitial spaces and around larger bronchioles. CD4+ lymphocytes could be seen in aggregates around small vessels, some of which appeared to contain platelets and small thrombi. The alveolar capillaries were remarkably thickened, with edema in the surrounding and fibrin thrombi within the capillaries and small vessels. CD61+ megakaryocytes possibly representing resident pulmonary megakaryocytes, with significant nuclear atypia and hyperchromasia were evident. The fibrin and platelets present within small vessels also appeared to aggregate inflammatory cells, with entrapment of numerous neutrophils. Only in one immunosupressed patient, there was an evidence of focal acute inflammatory infiltrate possibly consistent with secondary infection and were present in association with clusters of CD4+ mononuclear cells [9]. Zhexu et al showed bilateral DAD along with cellular fibromyxoid exudates. There was evidence of desquamation of pneumocytes and hyaline membrane formation, which indicated acute respiratory distress syndrome. The lungs revealed pulmonary oedema with hyaline membrane formation which was suggestive of earlyphase ARDS. Interstitial mononuclear inflammatory cell infiltrate, dominated by lymphocytes, were seen in both lungs. B Henley et al in their study on 50-year- old man, showed DAD with exudates formation. The inflammatory cells comprised predominantly of lymphocytes and multinucleated giant cells were seen along with large atypical pneumocytes. Carsana et al in their study also showed the features of DAD corresponding to those observable in the exudative and early/intermediate proliferative phases of the disease. Both phases often overlapped in the different areas of the lungs, with plurifocal pattern of distribution. Moreover, few patients also had bacterial and fungal abscesses formation [10]

Tien et al in their study of coronavirus through post mortem biopsies of liver stated that the liver showed only mild sinusoidal dilatation, which is a common finding in terminally ill patients. Focal macro vesicular steatosis, nuclear glycogen accumulation in hepatocytes and dense atypical small lymphocytes in portal tracts was also seen [8].

Fox et al stated that Cardiomegaly was the most common gross finding with right ventricular dilatation. On histopathological examination, heart was unremarkable; however, for scattered myocyte necrosis was evident in all speciemens. Few areas revealed lymphocytes adjacent to degenerating myocytes (but not surrounding). Whether this was an early manifestation of a viral myocarditis is not known, but there was no significant lymphocytic inflammatory infiltrate that was found to be consistent with the typical pattern of viral myocarditis [9]. Chen et al hypothesizesed that pericytes may be infected by the virus and it can cause capillary endothelial cell/microvascular dysfunction which may cause individual cell necrosis. There was no clear viral cytopathic effect found on light microscopy, but the possibility of direct viral infection of myocytes cannot be entirely ruled [6]. Tian S et al studied cardiac biopsies in two cases and found that their endocardia and myocardia did not show any signs of inflammation. Although, focally, the myocardium seems to be abnormal with presence of dark cytoplasm, these modifications were not sufficient for interpretation of acute myocardial injury. There were various findings, which included, 
focal edema, interstitial fibrosis and myocardial hypertrophy. These latter findings probable constitute pre-existing modifications related to patients underlying diseases, together with hypertension-related myocardial hypertrophy and past ischemic injury [8].

$\mathrm{Su}$ et al in their study on post mortem findings of renal changes in patients of COVID-19 stated hypoxia, abnormal coagulation and rhabdomyolysis are the factors contributing to renal injury in these patients. Only 9 patients had a pre-morbid urinalysis. Seven of nine presented cases had proteinuria and 6 of 9 had haematuria. Two patients had acute pyelonephritis and 5 patients $(19 \%)$ required continuous renal replacement therapy. In Kidney pathology of COVID-19 autopsies all reported cases were found to have mild to severe acute tubular injury. Loss of the proximal tubular brush borders was a characteristic feature of acute tubular injury with coagulative necrosis, vacuolar degeneration (non-isometric in most cases), (4 cases), hemosiderin granules within tubular cytoplasm and pigmented casts within tubular lumens. Deposition of hemosiderin was present in four cases. Evidence of glomerular ischemia was also seen. No significant finding was seen on Immunofluorescence except for a single reported case of IgA nephropathy. Fibrin tactoids or platelet aggregates were not observed in any COVID-19 cases. The receptor for SARS-CoV2, ACE2, was found to be overexpressed in parenchyma of kidney sections of COVID 19 cases. Positive immunofluorescence staining for SARS-CoV2 antibody within tubular epithelial cells was also present within 3 of 6 stained cases. In all decedents hypertensive-related vascular changes were seen and were found to be moderate to severe in 18 patients while platelet rich thrombi and red blood cell fragmentation were not identified in any case [7].

\section{CONCLUSION}

Medical autopsies are not commonly performed. Moreover, biosafety concerns arise in patients died of COVID-19. In the early phase of the outbreak, no autopsies were performed on patients dying of COVID19. Although, later autopsies were permitted under strict biosafety regulations. We recognize that due to limited sampling in needle core procedures, studies using postmortem biopsies carry certain limitations

\section{REFERENCES}

1. Report of clustering pneumonia of unknown etiology in Wuhan City. 2019. http://wjw.wuhan.gov.cn/front/web/showDetail/2019123108989.

2. Chen Y,Liu Q, Guo D. Coronaviruses: genome structure, replication and pathogenesis. J Med Virol. 2020:jmv.25681.

3. Li W. Bats are natural reservoirs of SARS-like coronaviruses. Science.2005; 310(5748):676-9.

4. Kupferschmidt K. Emerging diseases. Researchers scramble to understand camel connection to MERS. Science. 2013;341(6147):702.

5. Zhu N, Zhang D, Wang W et al. A novel coronavirus from patients with pneumonia in China, 2019. NEngl JMed. 2020. https://doi.org/10.1056/NEJMoa2001017

6. Chan JFW, Yuan S, Kok KH et al. A familial cluster of pneumonia associated with the 2019 novel coronavirus indicatingperson`to"person transmission: a study of a family cluster. Lancet.2020; 395:514-23.

7. Su H, Yang M, Wan C, Yi LX, Tang F, Zhu Hyd et al. Renal histopathological analysis of 26 postmortem findings of patients with COVID-19 in China. Kidney International. 2020 Apr 9.

8. Tian S, Xiong Y, Liu H, Niu L, Guo J, Liao M et al. Pathological study of the 2019 novel coronavirus disease (COVID-19) through postmortem core biopsies. Modern Pathology. 2020 Apr 14:1-8.

9. Fox SE, Akmatbekov A, Harbert JL, Li G, Brown JQ, Vander Heide RS. Pulmonary and Cardiac Pathology in Covid-19: The First Autopsy Series from New Orleans. medRxiv. 2020 Jan 1.

10. Zhexu Z, Shi L, Wang Y, Zhang J, Huang L, Zhang C. Pathological findings of COVID19 associated with acute respiratory distress syndrome. The Lancet respiratory medicine. 2020 Apr 1;8(4):420-2.

11. Hanley B, Lucas SB, Youd E, Swift B, Osborn M. Autopsy in suspected COVID-19 cases. Journal of Clinical Pathology. 2020 May 1;73(5):239-42.

12. Carsana L, Sonzogni A, Nasr A, Rossi R, Pellegrinelli A, Zerbi P et al. Pulmonary postmortem findings in a large series of COVID-19 cases from Northern Italy. medRxiv. 2020 Jan 1. 